\title{
Effects of the Radial Electric Field on the Confinement of Trapped Fast Ions in Wendelstein 7-X and Helias reactor
}

\author{
Ya.I. Kolesnichenko, V.V. Lutsenko, A.V. Tykhyy \\ Institute for Nuclear Research, Prospekt Nauky 47, Kyiv 03680, Ukraine* \\ A. Weller, A. Werner, H. Wobig, J. Geiger \\ Max-Planck-Institut für Plasmaphysik, \\ IPP-EURATOM Association, D-17489 Greifswald, Germany
}

(Dated: April 6, 2006)

Confinement of trapped fast ions in the Wendelstein-line stellarators in the presence of the radial electric field, $E_{r}$, is studied. It is shown that negative electric field improves the confinement; in particular, a radially localized field can play the role of a transport barrier for the ions escaping from the plasma when $E_{r}=0$. In contrast to this, positive electric field tends to deteriorate the ion confinement, unless its magnitude is very large. Such a field accompanied by the plasma rotation with the frequency around a certain magnitude, which we refer to as the resonance rotation frequency, leads to quick particle loss. A possibility to use the plasma rotation with the resonance frequency for the ash removal in a Helias reactor is considered. The mentioned results are obtained analytically and numerically. The analytical consideration was done on the basis of the derived bounce-averaged equations of the particle motion. The numerical calculations were carried out for Wendelstein 7-X [G. Grieger et al., J. Plasma Fusion Res. SERIES 1, 53 (1998)] and a Helias reactor [J. Kisslinger et al., Proc. 17th Int. Conf. (Yokohama, 1998) vol. 4, 1239 (1999)] by the guiding center code ORBIS (ORBits In Stellarators) developed in this work.

PACS numbers: 52.50.Gj, 52.55.Hc, 52.65.Cc, 02.60.Cb

Keywords: Stellarator, Electric field, Fast Ions

${ }^{*}$ Electronic address: yk@nucresi.freenet.kiev.ua 


\section{INTRODUCTION}

There will be several sources of fast ions in the Wendelstein 7-X (W7-X) stellarator. ${ }^{1}$ They are the Neutral Beam Injection (NBI), Ion Cyclotron Resonance Heating (ICRH), and Negative-ion-based Neutral Beam Injection (NNBI) at the later stage of operation, which will provide the plasma heating. ${ }^{2}$ In addition, it is planned to install an NBI injector for the diagnostics. ${ }^{3}$ Some energetic ions will be produced due to fusion reactions, mainly due to beam-plasma interaction. On the other hand, an important source of fast ions in reactor plasmas, in particular, in a Helias reactor ${ }^{4}$ will be a thermonuclear reaction, which will produce both circulating and trapped particles. In contrast to this, NBI in W7-X will produce mainly circulating (marginally circulating) particles. Nevertheless, a considerable amount of the trapped fast ions will be present in W-7X, at least, in regimes with the temperature $T>2 \mathrm{keV}$ and $z_{\text {eff }}>1\left(z_{\text {eff }}\right.$ is the effective charge number $)$ because in these regimes the energy of injected ions, $\mathcal{E}_{b} \lesssim 60 \mathrm{keV}$, is about the energy $\mathcal{E}_{*} \propto\left(M_{i} / M_{e}\right)^{1 / 3} T$ ( $M_{i / e}$ is the ion/electron mass) for which $\tau_{\perp} \sim \tau_{s}$, where $\tau_{\perp}$ and $\tau_{s}$ are the characteristic times of Coulomb pitch-angle scattering and slowing down, respectively. In addition, trapped particles will be directly produced by the diagnostic NBI and ICRH. Thus, fast ions with various energies and pitch-angles will be present in plasmas of both W7-X and a Helias reactor.

The basic idea to provide good confinement of the trapped fast ions in the Wendelsteinline stellarators is to achieve a sufficiently high $\beta$ (the ratio of the plasma pressure to the magnetic field pressure), $\beta \sim 5 \% .^{5}$ At high $\beta$ the plasma diamagnetism "kills" unclosed superbanana orbits by making contours of the longitudinal adiabatic invariant $\left(J_{\|}=\oint v_{\|} d l\right)$ closed and weakly deflecting from the magnetic flux surfaces. On the other hand, the radial electric field, $E_{r}$, also affects the particle confinement. However, in contrast to the plasma diamagnetism, the electric field effects are important only for particles with the energy less than a certain magnitude determined from the condition that the electric drift velocity is about or more than the magnetic drift velocity. In addition, electric field effects depend on the sign of the electric field.

Note that the radial electric field is always present in the plasma. A simple estimate based on the assumption that $e \Phi \sim T=2-10 \mathrm{keV}$ ( $\Phi$ is the scalar potential of the electric field) leads to $E_{r} \sim 4-20 \mathrm{kV} / \mathrm{m}$ in a plasma with the radius $a=0.5 \mathrm{~m}$. In reality, the electric 
field can be even stronger. Its magnitude is determined by many factors, such as transport properties of the bulk plasma, plasma parameters and their radial profiles, the confinement of fast ions, etc. The electric field $\left|E_{r}\right|=20 \mathrm{kV} / \mathrm{m}$ was generated during perpendicular NBI in Wendelstein 7 -AS. ${ }^{6}$ Neoclassical calculations predict $E_{r} \lesssim 18 \mathrm{kV} / \mathrm{m}$ in the plasma core (the electron root) and $\left|E_{r}\right| \lesssim 5 \mathrm{kV} / \mathrm{m}$ with $E_{r}<0$ (the ion root) at the periphery for a particular case in Wendelstein $7-\mathrm{X} .{ }^{7}$

The main purpose of this work is to study effects of the electric field on the confinement of trapped fast ions in the Wendelstein-line stellarators, namely, in W7-X and a Helias reactor. The structure of the work is as follows. In section II equations of the particle motion in the general stellarator magnetic field in the presence of the equilibrium electric field are derived; a code ORBIS solving the derived equations is described. In section III the bounce averaged equations of the motion of the locally trapped particles are obtained and analyzed qualitatively by keeping several Fourier harmonics, which dominate in the magnetic field of the Wendelstein-line stellarators. In section IV the confinement of fast ions in W7-X is studied numerically by the code ORBIS. In section $\mathrm{V}$ a possibility to use the radial electric field for the ash removal in a Helias reactor is considered. At last, in section VI the obtained results are summarized.

\section{BASIC EQUATIONS. CODE ORBIS.}

The motion of the particle guiding center in a steady-state magnetic field, $\mathbf{B}$, and a potential electric field, $\mathbf{E}=-\nabla \Phi$ with $\Phi$ the scalar potential, is described by the following equations:

$$
\begin{gathered}
\dot{\mathbf{r}}_{g c}=\mathbf{v}_{\|}+\mathbf{v}_{D}+\mathbf{v}_{E}, \\
\dot{\rho}_{\|}=\left(\mathbf{v}_{\|}+\mathbf{v}_{D}+\mathbf{v}_{E}\right) \cdot \nabla \rho_{\|},
\end{gathered}
$$

where $\dot{\mathbf{r}}_{g c}=d \mathbf{r}_{g c} / d t, \dot{\rho}_{\|}=d \rho_{\|} / d t, \mathbf{r}_{g c}$ is the radius-vector of the particle guiding center, $\rho_{\|}=v_{\|} / \omega_{B}, v_{\|}=\mathbf{v} \cdot \mathbf{b}$ is the velocity along the magnetic field, $\mathbf{b}=\mathbf{B} / B, \mathbf{B} \equiv \mathbf{B}\left(\mathbf{r}_{g c}\right)$,

$$
v_{\|}=\sigma \sqrt{\frac{2}{M}\left[W-e \Phi-\mu_{p} B\right]}, \quad W=\mathcal{E}+e \Phi, \quad \frac{d W}{d t}=0
$$


$\mu_{p}$ is the particle magnetic moment, $\mathcal{E}=M v^{2} / 2$ is the particle kinetic energy, $e$ is the particle charge, $\sigma=v_{\|} /\left|v_{\|}\right|, \mathbf{v}_{D}$ and $\mathbf{v}_{E}$ are the drift velocities given by

$$
\mathbf{v}_{D}=\frac{1}{\omega_{B}} \mathbf{b} \times\left(\frac{\mu_{p}}{M} \nabla B+v_{\|}^{2} \mathcal{K}\right), \quad \mathbf{v}_{E}=c \frac{\mathbf{E} \times \mathbf{B}}{B^{2}},
$$

$\mathcal{K}=B^{-2}\left(B \nabla_{\perp} B+4 \pi \nabla_{\perp} p\right)$ is the field line curvature, $p$ is the plasma pressure, $\omega_{B}$ is the particle gyrofrequency.

Noting that $\nabla v_{\|}=\left(e \mathbf{E}-\mu_{p} \nabla B\right) / M v_{\|}$, it is easy to show that the terms proportional to the electric field in the right-hand-side of Eq. (2) can be written as follows (we will label them by the superscript "E"):

$$
\left(\dot{\rho}_{\|}\right)^{E}=\left(\dot{\mathbf{r}}_{g c} \cdot \nabla \rho_{\|}\right)^{E}=\frac{c E_{\|}}{B}+\frac{4 \pi c v_{\|}}{\omega_{B}|B|^{4}} \mathbf{E} \cdot[\mathbf{B} \times \nabla p] .
$$

We assume that $\Phi=\Phi(r)$, where $r$ is a the radial flux coordinate. Then it follows from Eq. (5) that $\left(\dot{\mathbf{r}}_{g c} \cdot \nabla \rho_{\|}\right)^{E}=0$. This implies that the equation for $\rho_{\|}$in the presence of the electric field can be written in the form coinciding with that in the absence of the electric field in the considered case of $\Phi=\Phi(r)$. Therefore, we will use this equation rather than the equation for $v_{\|}$[which depends explicitly on the electric field because $\left(\dot{v}_{\|}\right)^{E}=$ $\left.v_{\|} \mathbf{v}_{E} \cdot \nabla_{\perp} B / B \neq 0\right]$.

Below we use the flux coordinates $r, \vartheta, \varphi$, where $r$ is defined by $\psi=\bar{B} r^{2} / 2$, with $\psi$ the toroidal magnetic flux, and $\bar{B}$ the average magnetic field at the magnetic axis; $\psi, \vartheta$ and $\varphi$ being the Boozer coordinates. Then, assuming $\beta \ll 1$ and $N \iota r^{2} / R_{0}^{2} \ll 1$ ( $N$ is the number of the field periods, $\iota$ is the rotational transform, $R_{0}$ is the large radius of the torus) we can write Eqs. (1), (2) in the following form:

$$
\begin{gathered}
\dot{r}=-\frac{v_{d}}{\epsilon} \frac{1}{B} \frac{\partial B}{\partial \vartheta}, \\
\dot{\vartheta}=v_{\|} \iota b^{3}+\frac{v_{d}}{\epsilon} \frac{1}{B} \frac{\partial B}{\partial r}+\Omega_{E}, \\
\dot{\varphi}=v_{\|} b^{3}, \\
\dot{\rho}_{\|}=-\frac{v_{d}}{B}\left(\frac{\partial B}{\partial \varphi}+\iota \frac{\partial B}{\partial \vartheta}\right),
\end{gathered}
$$


where $v_{\|}=\rho_{\|} \omega_{B 0} B / \bar{B}, \omega_{B 0}=e \bar{B} /(M c), \Omega_{E}=-c E_{1} /(\bar{B} r)$ is the frequency of the electricfield-induced motion in the poloidal direction, $\iota=B^{2} / B^{3}$, the superscripts and subscripts " $1,2,3$ " denote the contra-variant and co-variant vector components, respectively, $\epsilon=r / R_{0}$, $v_{d}$ is the "tokamak" drift velocity given by

$$
v_{d}=\frac{\left(v^{2}+v_{\|}^{2}\right)}{2 \omega_{B 0} R_{0}}=\left(\rho_{\|}^{2}+\frac{\lambda \mathcal{E}_{0} \bar{B}}{M \omega_{B 0}^{2} B}\right) \frac{\omega_{B 0}|B|^{2}}{R_{0}|\bar{B}|^{2}},
$$

$\lambda=\mu_{p} \bar{B} / \mathcal{E}_{0}$ is an initial pitch-angle parameter, $\mathcal{E}_{0} \equiv \mathcal{E}(t=0), b^{3} \approx B /\left(\bar{B} R_{0}\right)$. Equations (6)-(8) neglect the influence of small components of the magnetic field, $B_{1}$ and $B_{2}$, on the particle drift motion. Note that $\lambda$ is connected with the initial pitch angle, $\chi_{0}=v_{\|} /\left.v\right|_{t=0}$ as follows: $\lambda=\left(1-\chi_{0}^{2}\right) \bar{B} / B\left(\mathbf{r}_{0}\right)$ with $\mathbf{r}_{0} \equiv \mathbf{r}(t=0)$.

Equations (6)-(10) were used to develop the code ORBIS (ORBits In Stellarators). The normalized radial coordinate $\widetilde{r}=r / a$, with $a$ the average plasma radius defined by $\psi_{a}=$ $\bar{B} a^{2} / 2$, was introduced and the magnetic field strength was taken in the form

$$
B=\sum_{\mu \geq 0, \nu} B^{(\mu \nu)}(\widetilde{r}) \cos (\mu \vartheta-\nu N \varphi)
$$

where $\mu$ and $\nu$ are the poloidal and toroidal numbers of the magnetic field, respectively. The code ORBIS solves the initial value problem. It uses equilibrium data as the input. The output is, first, the particle orbits in Boozer coordinates and in real coordinates, and second, the magnetic field along the particle orbits. Graphical presentation of the particle motion in both the poloidal plasma cross-section and toroidal cross-section is possible. The ability of ORBIS to visualize on the PC monitor the particle trajectory as a function of time is to be used in the "virtual" (numerical) W7-X stellarator, ${ }^{8}$ which was an original motivation for the development of this code.

In many cases, it is convenient to present the magnetic field as follows:

$$
B=\bar{B}\left[1+\sum_{\mu \geq 0, \nu} \epsilon_{B}^{(\mu \nu)}(r) \cos (\mu \vartheta-\nu N \varphi)\right],
$$

Then Eqs. (6)-(9) take the form:

$$
\dot{r}=\frac{v_{d} R_{0}}{r} \frac{\bar{B}}{B} \sum_{\mu \nu \neq 0} \mu \epsilon_{B}^{(\mu \nu)} \sin (\mu \vartheta-\nu N \varphi),
$$




$$
\begin{gathered}
\dot{\vartheta}=\iota \omega_{B 0} \frac{\rho_{\|}}{R_{0}}\left(\frac{B}{\bar{B}}\right)^{2}+\frac{v_{d} R_{0}}{r} \frac{\bar{B}}{B} \sum_{\mu \geq 0, \nu} \frac{d \epsilon_{B}^{(\mu \nu)}}{d r} \cos (\mu \vartheta-\nu N \varphi)+\Omega_{E}, \\
\dot{\varphi}=\omega_{B 0} \frac{\rho_{\|}}{R_{0}}\left(\frac{B}{\bar{B}}\right)^{2}, \\
\dot{\rho}_{\|}=v_{d} \frac{\bar{B}}{B} \sum_{\mu \nu \neq 0}(\mu \iota-\nu N) \epsilon_{B}^{(\mu \nu)} \sin (\mu \vartheta-\nu N \varphi) .
\end{gathered}
$$

To choose the initial $\rho_{\|}$the following equation can be used:

$$
\rho_{\|}(t=0)=\left.\frac{\sigma \bar{B}}{B \omega_{B 0}} \sqrt{\frac{2 \mathcal{E}}{M}} \sqrt{1-\frac{\lambda B}{\bar{B}}}\right|_{t=0} .
$$

\section{BOUNCE-AVERAGED EQUATIONS AND THEIR ANALYSIS}

The equations derived in the previous section can be solved only numerically. However, in many cases analytical consideration and qualitative analysis are useful. Therefore, below we derive equations describing average particle motion. This can be done due to the fact that $N \gg 1$, whereas $\mu \leq 1$ and $\nu \leq 1$, which leads to two scales of the modulation of $B$ along the field lines, $\Delta \varphi \sim \pi / N$ and $\Delta \varphi \sim \pi$, see Fig. 2 .

In many stellarators dominant harmonics of the magnetic field strength have the same magnitude of $\nu \neq 0$. In this case Eq. (12) is reduced to (cf. Ref. ${ }^{9}$ )

$$
B=\bar{B}\left\{1+\epsilon_{0}(r)-\epsilon_{t}(r) \cos \vartheta+\epsilon_{H}(r, \vartheta) \cos [N \varphi-\xi(r, \vartheta)]\right\}
$$

where $\epsilon_{0} \equiv \epsilon_{B}^{(00)}>0$ is the diamagnetic harmonic, $\epsilon_{t} \equiv-\epsilon_{B}^{(10)}>0$ is associated with the toroidicity (but $\epsilon_{t} \neq r / R_{0} !$ ),

$$
\begin{gathered}
\epsilon_{H}=\frac{a_{1}}{\left|a_{1}\right|} \sqrt{a_{1}^{2}+b_{1}^{2}}, \quad \xi=\cos ^{-1} \frac{a_{1}}{\epsilon_{H}}=\operatorname{tg}^{-1} \frac{b_{1}}{a_{1}}, \\
a_{1}=\sum_{\mu} \epsilon^{(\mu \hat{\nu})} \cos \mu \vartheta, \quad b_{1}=\sum_{\mu} \epsilon^{(\mu \hat{\nu})} \sin \mu \vartheta,
\end{gathered}
$$

$\hat{\nu}$ is the toroidal number of dominant $\nu \neq 0$ harmonics. In particular, $\hat{\nu}=1$, and the harmonics with $\mu=0,1$ mainly contribute in the Wendelstein-line stellarators, see Fig. 1, which leads to 


$$
\epsilon_{H}=\sqrt{\epsilon_{m}^{2}+\epsilon_{h}^{2}-2 \epsilon_{m} \epsilon_{h} \cos \vartheta}, \quad \cos \xi=\frac{\epsilon_{m}-\epsilon_{h} \cos \vartheta}{\epsilon_{H}},
$$

where $\epsilon_{m}=\epsilon^{(01)}>0$ and $\epsilon_{h}=-\epsilon^{(11)}>0$ are the amplitudes of the mirror harmonic and helical harmonic, respectively.

As we already mentioned, there are to scales of the modulation of $B$. Therefore, the particle motion can be presented as a superposition of slow motion and fast motion. Then Eqs. (13)-(16) can be averaged over the fast motion. Taking the magnetic field in the form given by Eq. (18), we obtain the following bounce averaged equations for the locally trapped particles:

$$
\begin{gathered}
\langle\dot{r}\rangle=\frac{\bar{v}_{d}}{\epsilon}\left[\frac{\partial \epsilon_{H}}{\partial \vartheta}\left(\frac{2 E(\kappa)}{K(\kappa)}-1\right)-\epsilon_{t} \sin \vartheta\right] \\
\langle\dot{\vartheta}\rangle=\frac{\bar{v}_{d}}{\epsilon}\left[-\frac{\partial \epsilon_{H}}{\partial r}\left(\frac{2 E(\kappa)}{K(\kappa)}-1\right)-\frac{\partial \epsilon_{t}}{\partial r} \cos \vartheta+\frac{\partial \epsilon_{0}}{\partial r}\right]+\Omega_{E}, \\
\langle\dot{\varphi}\rangle=\frac{1}{N} \frac{\partial \xi}{\partial \vartheta}\langle\dot{\vartheta}\rangle+\frac{1}{N} \frac{\partial \xi}{\partial r}\langle\dot{r}\rangle,
\end{gathered}
$$

where $\bar{v}_{d}=v_{d}\left(v_{\|}=0\right), K=K(\kappa)$ and $E=E(\kappa)$ are the complete elliptical integrals of the first kind and second kind, respectively,

$$
\begin{gathered}
\kappa^{2}=\frac{1}{2}+\frac{\alpha-e \Phi /\left(\mu_{p} \bar{B}\right)-\epsilon_{0}+\epsilon_{t} \cos \vartheta}{2\left|\epsilon_{H}\right|}, \quad \alpha=\frac{W}{\mu_{p} \bar{B}}-1, \\
\frac{\partial \epsilon_{H}}{\partial r}=\frac{\epsilon_{h}-\epsilon_{m} \cos \vartheta}{\epsilon_{H}} \frac{\partial \epsilon_{h}}{\partial r}+\frac{\epsilon_{m}-\epsilon_{h} \cos \vartheta}{\epsilon_{H}} \frac{\partial \epsilon_{m}}{\partial r}, \\
\frac{\partial \epsilon_{H}}{\partial \vartheta}=\frac{\epsilon_{m} \epsilon_{h}}{\epsilon_{H}} \sin \vartheta .
\end{gathered}
$$

Equations (22), (23) do not contain the toroidal coordinate and are sufficient to describe the orbits in the $(r, \vartheta)$ plane. Equation $(24)$ for $\langle\dot{\varphi}\rangle$ is obtained in the assumption that $N \varphi-\xi=$ const, which is true when $\epsilon_{t} \ll \max \left(\epsilon_{m}, \epsilon_{h}\right)$.

Note that locally trapped particles oscillate around the points where the magnetic field along the field line, $B(s)$, has minima (in these points $\kappa^{2}=0$ implies $v_{\|}=0$ ). In particular, in the case when the mirror harmonic of $B$ exceeds other harmonics, $B(s)$ has minima at the outer circumference of the torus $(\vartheta=0)$ at $\varphi=(2 n+1) \pi / N$ with $n$ the integer. On the 
other hand, the maxima for $\vartheta=0$ are located at $\varphi=2 n \pi / N\left(\right.$ where $\kappa^{2}=1$ for $\left.v_{\|}=0\right)$. In this case the particles injected with small pitch angles at $\vartheta=0, \varphi=2 n \pi / N$ will be either marginally locally trapped or locally passing.

Locally trapped particles and completely passing particles have $\kappa_{\max }<1$ and $\kappa_{\min }>1$, respectively, where $\kappa_{\max / \min }$ is the maximum/minimum magnitude of $\kappa(r, \vartheta)$ on the particle orbit $[\kappa(r, \vartheta)$ is not a constant of motion]. Particles crossing the separatrix in the phase space between the regions of the locally trapped particles and locally passing ones (where $\kappa=1$ ) during their orbital motion are transitioning. Taking this into account and assuming that the particle orbit width $(\Delta r)$ is small, we obtain from the Eq. (25) the following conditions for the pitch parameter, $\alpha$, of various groups of the particles:

$$
\begin{gathered}
\alpha_{\text {min }}<\alpha^{l o c}<\epsilon_{0}+\epsilon_{m}-\epsilon_{h}-\epsilon_{t}, \\
\epsilon_{0}+\epsilon_{m}-\epsilon_{h}-\epsilon_{t}<\alpha^{\text {tran }}<\epsilon_{0}+\epsilon_{m}+\epsilon_{h}+\epsilon_{t}, \\
\alpha^{\text {pass }}>\epsilon_{0}+\epsilon_{m}+\epsilon_{h}+\epsilon_{t},
\end{gathered}
$$

where $\alpha^{l o c}, \alpha^{\text {tran }}$, and $\alpha^{\text {pass }}$ are the pitch-angle parameters of the localized particles, transitioning particles, and passing particles, respectively, $\alpha_{\min }$ is determined by the equation $\kappa(r, \vartheta)=0$. In particular, $\alpha_{\text {min }}=\epsilon_{0}-\epsilon_{m}+\epsilon_{h}-\epsilon_{t}$ for $\vartheta=0$ and $\alpha_{\min }=\epsilon_{0}-\epsilon_{m}-\epsilon_{h}+\epsilon_{t}$ for $\vartheta=\pi$. The electric potential, $\Phi$, was taken zero in Eqs. (28)-(30), which is justified due to the used assumption that $\Delta r$ is small. When $e E_{r} \Delta r / \mathcal{E} \gtrsim \epsilon_{t}$, the electric field considerably affects the conditions given by Eqs. (28)-(30).

Let us take into account that $\epsilon_{h}$ and $\epsilon_{t}$ are approximately proportional to $r$. In addition, let us neglect the terms proportional to $\epsilon_{m}^{\prime}$ in Eq. (26). One can see that the last term in Eq. (26) is indeed small due to fulfilment of the inequality $d \ln \epsilon_{m} / d \ln r \ll 1$, whereas the previous term is negligible when $r \epsilon_{m} d \epsilon_{m} / d r \ll \epsilon_{h}^{2}$, which is the case at low $\beta$ only (the derivative of $\epsilon_{m}$ grows with $\beta$ ). In spite of this fact, our approximation leads to results which are in a reasonable agreement with the numerical results obtained by the code ORBIS, as will be shown below. In addition, this approximation will enable us to obtain a general qualitative picture of the influence of the radial electric field on the confinement of fast ions. 
Making the mentioned simplifying assumptions, we can approximate Eqs. (22), (23) as follows:

$$
\langle\dot{r}\rangle=u \sin \vartheta
$$

$$
r\langle\dot{\vartheta}\rangle=u \cos \vartheta+w
$$

where

$$
\begin{gathered}
u=\frac{\bar{v}_{d}}{\epsilon}\left[\frac{\epsilon_{m} \epsilon_{h}}{\epsilon_{H}}\left(\frac{2 E(\kappa)}{K(\kappa)}-1\right)-\epsilon_{t}\right], \\
w=\frac{\bar{v}_{d}}{\epsilon}\left[r \epsilon_{0}^{\prime}-\frac{\epsilon_{h}^{2}}{\epsilon_{H}}\left(\frac{2 E(\kappa)}{K(\kappa)}-1\right)\right]+v_{E},
\end{gathered}
$$

"prime" means the radial derivative, $v_{E}=r \Omega_{E}$, and, in general, $u=u(r, \vartheta), w=w(r, \vartheta)$.

One can see that when

$$
\delta \equiv\left|\frac{w}{u}\right| \ll 1
$$

Eqs. (31), (32) yield $x \equiv r \cos \vartheta \approx$ const, which means that the particles are not confined. In the contrary case, $\delta \gg 1$, the particles are well confined, at least, when $u$ and $w$ weakly depend on $\vartheta$. It is sufficient to require $\delta \gg 1$ only in the plasma periphery in order to provide the particle confinement. It is clear that $\delta \rightarrow \infty$ for $u \rightarrow 0$, i.e., when the radial drift vanishes. In particular, in stellarators with $\epsilon_{m} \gg \epsilon_{h}$, the radial drift of the well trapped particles is minimum when

$$
\epsilon_{h}=\epsilon_{t}
$$

Let us first assume that the radial electric field is absent and the plasma pressure is negligible $\left(\epsilon_{0}^{\prime} \approx 0\right)$. Then

$$
\delta=\left|\frac{\frac{\epsilon_{h}^{2}}{\epsilon_{H}}\left(\frac{2 E(\kappa)}{K(\kappa)}-1\right)}{\frac{\epsilon_{m} \epsilon_{h}}{\epsilon_{H}}\left(\frac{2 E(\kappa)}{K(\kappa)}-1\right)-\epsilon_{t}}\right| .
$$

It follows from Eq. (37) that $\delta \ll 1$ for the marginally trapped particles with $\kappa \sim 0.9$. However, because $\kappa=\kappa(r, \vartheta), \kappa$ changes during the orbital motion and, therefore, the particles with $\kappa \sim 0.9$ are not necessarily lost. When the trapping parameter continues to grow 
after reaching the magnitude of 0.9 , the orbit transformation into the locally passing state may occur and the stochastic diffusion may take place. ${ }^{9}$ The behaviour of the well-trapped particles $(\kappa \ll 1)$ and marginally trapped particles $(\kappa \rightarrow 1)$ depends on the relationships between the harmonics of the magnetic field. In particular, for a configuration with the dominant mirror harmonic [the high-mirror configuration, $\epsilon_{m} \gg \max \left(\epsilon_{h}, \epsilon_{t}\right)$ ] we obtain from Eq. (37) that $|\delta| \sim \epsilon_{h} / \epsilon_{m} \ll 1$, i.e., the particles are not confined. In the contrary case, when the mirror harmonic is small, $\delta \sim\left(\epsilon_{h} / \epsilon_{t}\right)|2 E(\kappa) / K(\kappa)-1|, \delta>1$ for $\epsilon_{h}>\epsilon_{t}$ unless $\kappa \sim 0.9$, in which case a particle moves along the superbanana orbit with the orbit width $\Delta r \sim r \sqrt{\epsilon_{t} / \epsilon_{h}} \cdot 10$

Finite plasma pressure can provide the confinement of the localized particles in the highmirror configuration. For the well-localized particles $(\kappa \rightarrow 0)$ this will be the case when $r \epsilon_{0}^{\prime} / \epsilon_{h} \gg 1$ (then $\delta \gg 1$ ). A milder condition is required for the confinement of particles with finite $\kappa$. Taking $\epsilon_{h} \propto \epsilon_{t} \propto \epsilon, \epsilon_{0} \propto r^{2}$ we conclude that $u \approx$ const and $\delta \propto r$ for the well-localized particles. Then we obtain that the particle orbits described by Eqs. (31), (32) are given by

$$
x-x_{0}=\frac{\delta_{1}}{2 a}\left(r_{0}^{2}-r^{2}\right)
$$

with $\delta_{1}$ defined by $\delta=\delta_{1} r / a,\left(r_{0}, \vartheta_{0}\right)$ is a point on the particle orbit. This yields the orbit width $\Delta r / a=2 / \delta_{1}$. Although Eq. (38) was obtained for $E_{r}=0$, it is also valid in the presence of the electric field provided that $E_{r} \propto r$. Moreover, on a qualitative level, this equation can be used for an arbitrary electric field [when $E_{r}$ is small, it can be neglected; when $E_{r}$ is so large that $\delta \gg 1$, Eq. (38) predicts the particle motion along the $r=$ const surfaces, which is true for any dependence of $E_{r}$ of $r$ ].

Now we proceed to analysis of effects of the electric field. First of all, we note that the signs of the $\epsilon_{0}^{\prime}$ term and the E-field term in Eq. (32) are the same when $E_{r}<0$. Therefore, the negative radial electric field can provide the confinement of the trapped particles in high-mirror configurations when $\beta$ is not sufficiently large for the particle confinement at $E_{r}=0$. The required magnitude of the electric field can be evaluated from the condition $\left|v_{E}\right| / u \gg 1$, which leads to

$$
\left.\left|E_{r}\left(r_{*}\right)\right| \gg \frac{\mathcal{E}\left(\epsilon_{h}-\epsilon_{t}\right)}{e r}\right|_{r=r_{*}}
$$


where $r_{*}$ is the radius where the electric field is maximum and $\epsilon_{h} \neq \epsilon_{t}$. The restriction $\epsilon_{h} \neq \epsilon_{t}$ is a consequence of the used approximation $\epsilon_{H} \approx \epsilon_{m}$ and the assumption $\kappa=0$ made when deriving Eq. (39). This approximation and neglecting finite $\kappa$ result in $u \propto \epsilon_{h}-\epsilon_{t}$, which is wrong when $\epsilon_{h}=\epsilon_{t}$. In the case when $e \Phi \sim T$, Eq. (39) can be written as a condition for the particle energy. We obtain:

$$
\mathcal{E} \ll \frac{T}{\epsilon_{h}-\epsilon_{t}} .
$$

Note that when the electric-field term dominates in Eq. (34), Eq. (40) represents a condition of the particle confinement independently on the sign of the electric field.

Below we show that the presence of the electric field in the narrow ring region, $\Delta_{E}$, can be sufficient to prevent the escape of the particles from the plasma. In other words, a radially localized negative electric field can play the role of a barrier for the fast ions. Such an electric field usually arises in turbulent plasmas with transport barriers and/or when the ion root of the neoclassical transport dominates in a part of the plasma cross section.

In order to provide the particle confinement, the width of the region where the electric field is localized, $\Delta_{E}$, must exceed $(\Delta r)_{E}$, where $(\Delta r)_{E}$ is the particle radial deflection in the electric-field region. Let us assume that an electric barrier is localized at the plasma periphery and a particle enter the barrier from the core region, so that a part of the orbit in the $(r, \vartheta)$-plane is located beyond the electric barrier. Then the particle radial deflection inside the barrier is less than $\Delta r$. Taking $(\Delta r)_{E} \sim \Delta r / 2$ with $\Delta r=2 a / \delta_{1}$ and assuming $\delta_{E} \sim \delta$ we can write the condition $\Delta_{E}>(\Delta r)_{E}$ as

$$
e\left|E_{r}\left(r_{*}\right)\right| \Delta_{E}>\left.\mathcal{E}\left(\epsilon_{h}-\epsilon_{t}\right)\right|_{r=r_{*}}
$$

where $\epsilon_{h} \neq \epsilon_{t}$. One can say that Eq. (41) represents a condition of the electric transport barrier for the trapped energetic ions.

In contrast to the negative electric field, the positive electric field competes with the finite pressure and can compensate the positive influence of high $\beta$ on the confinement of trapped particles in the high-mirror configuration. This takes place at a certain magnitude of the electric field,

$$
E_{r} \sim \bar{B} \frac{\bar{v}_{d}}{c} R_{0} \epsilon_{0}^{\prime} .
$$


When $E_{r} \sim \Phi / r \sim T /(e r)$, the energy of particles which are not confined because of the electric field compensating the effects of plasma diamagnetism can be evaluated as $\mathcal{E} \sim T /\left(r \epsilon_{0}^{\prime}\right)$.

According to Eq. (38), the worst confinement takes place when $\delta=0$. Then particles move along the orbits $x=$ const, which implies that $\Delta r=\infty$. Therefore, it is reasonable to introduce a resonance electric field, $E_{r}^{r e s}$, defined by the condition $\delta=0$. For the welltrapped particles we have:

$$
E_{r}^{r e s} \approx \bar{B} \frac{\bar{v}_{d}}{c \epsilon}\left[r \epsilon_{0}^{\prime}-\frac{\epsilon_{h}^{2}}{\epsilon_{H}}\right] .
$$

Because $E_{r}$ does not depend on $\vartheta$, this equation can be satisfied when either $\epsilon_{m} \gg \epsilon_{h}$ or $\epsilon_{m} \ll \epsilon_{h}$. When the second term in Eq. (43) exceeds the first one, the negative (rather than positive) electric field deteriorates the particle confinement. However, in this case the trapped energetic ions are not confined in systems with the dominant $\epsilon_{m}$ even in the absence of the electric field. Note that when $\epsilon_{0}=0, \epsilon_{m}=0$, Eq. (43) leads to the resonance radial electric field $E_{r}^{r e s} \sim-\epsilon_{h} \mathcal{E} /(e r)<0$ obtained in Ref. ${ }^{11}$.

When the mirror harmonic dominates in the magnetic field and the radial dependence of the diamagnetic component of the magnetic field can be approximated as $\epsilon_{0} \propto r^{2}$, the electric field given by Eq. (43) is proportional to $r$. This implies that this field leads to a rigid rotation of the plasma with the frequency

$$
\Omega_{E}^{r e s}(r)=-\omega_{B} \frac{\rho^{2} \epsilon_{0}}{r^{2}}\left(1-\frac{\epsilon_{h}^{2}}{2 \epsilon_{0} \epsilon_{m}}\right)=\text { const },
$$

where $\rho=v / \omega_{B}$. The width of the resonance can be evaluated by taking into account that particles are not confined when $\delta$ is small. Taking $\delta<\delta_{*}$ with $\delta_{*}>2$ at the plasma periphery, we evaluate the rotation frequency range for which the particles are not confined as follows:

$$
\Omega_{\min }<\left|\Omega_{E}\right|<\Omega_{\max }
$$

where

$$
\Omega_{\max / \min }=\omega_{B} \frac{\rho^{2}}{r^{2}}\left[\epsilon_{0}-\frac{\epsilon_{h}^{2}}{2 \epsilon_{m}} \pm \frac{\delta_{*}}{2}\left(\epsilon_{h}-\epsilon_{t}\right)\right]
$$


with $\epsilon_{h} \neq \epsilon_{t}$. If we require $\Omega_{E}(r)=$ const, we obtain $\delta_{*}=\delta_{* 1} r / a$, where $\delta_{* 1}$ can be considered as an adjustable parameter. Note that the relative width of the resonance does not depend on the particle energy:

$$
\frac{\Delta \Omega_{E}}{\Omega_{E}^{r e s}}=\frac{\delta_{*}\left(\epsilon_{h}-\epsilon_{t}\right)}{\epsilon_{0}-\frac{\epsilon_{h}^{2}}{2 \epsilon_{m}}}
$$

where $\Delta \Omega_{E}=\Omega_{\max }-\Omega_{\text {min }}$.

Equations (44)-(46) are written for particles with a given energy. In another case, when plasma rotation frequency is considered as a given quantity, these equations determine the resonance energy, $\mathcal{E}^{\text {res }}$, and the resonance width, $\Delta \mathcal{E}$, given by

$$
\begin{gathered}
\mathcal{E}^{r e s}=-\frac{M \omega_{B} \Omega_{E} r^{2}}{2\left(\epsilon_{0}-\frac{\epsilon_{h}^{2}}{2 \epsilon_{m}}\right)}, \\
\frac{\Delta \mathcal{E}}{\mathcal{E}^{r e s}}=\frac{\delta_{* 1}\left(\epsilon_{h}-\epsilon_{t}\right)\left(\epsilon_{0}-\frac{\epsilon_{h}^{2}}{2 \epsilon_{m}}\right) \frac{r}{a}}{\left(\epsilon_{0}-\frac{\epsilon_{h}^{2}}{2 \epsilon_{m}}\right)^{2}-\frac{\delta_{* 1}^{2}}{4}\left(\epsilon_{h}-\epsilon_{t}\right)^{2}}
\end{gathered}
$$

where $\Delta \mathcal{E}=\mathcal{E}_{\text {max }}-\mathcal{E}_{\text {min }}, \mathcal{E}_{\text {max } / \text { min }}$ is the maximum/minimum energy of particles affected by the resonance. It follows from Eqs. (47), (49) that the resonance width is proportional to $\epsilon_{h}-\epsilon_{t}$. However, it is not vanishing for $\epsilon_{h} \rightarrow \epsilon_{t}$, in which case finite $\kappa$ and the difference between $\epsilon_{H}$ and $\epsilon_{m}$ become important. The resonance width is not symmetric with respect to the resonance magnitude: $\mathcal{E}_{\text {max }}-\mathcal{E}^{\text {res }}>\left|\mathcal{E}_{\text {min }}-\mathcal{E}^{\text {res }}\right|$. This is seen from the equations

$$
\frac{\mathcal{E}_{\text {max } / \text { min }}}{\mathcal{E}^{\text {res }}}=\frac{\epsilon_{0}-\frac{\epsilon_{h}^{2}}{2 \epsilon_{m}}}{\epsilon_{0}-\frac{\epsilon_{h}^{2}}{2 \epsilon_{m}} \mp 0.5 \delta_{* 1}\left(\epsilon_{h}-\epsilon_{t}\right) \frac{r}{a}} .
$$

Equation (50) predicts $\mathcal{E}_{\text {max }}=\infty$ for $\left(\epsilon_{0}-0.5 \epsilon_{h}^{2} / \epsilon_{m}\right)=0.5 \delta_{* 1}\left(\epsilon_{h}-\epsilon_{t}\right) r / a$. This means that finite $\kappa$ and the difference between $\epsilon_{H}$ and $\epsilon_{m}$ must be taken into account in this case in order to obtain the correct magnitude of $\mathcal{E}_{\text {max }}$.

Note that the basic equations derived in this section [Eqs. (22), (23)] can also be obtained proceeding from the longitudinal invariant of the particle motion,

$$
J_{\|}=\oint v_{\|} d l=\frac{16 R_{0}}{N} \sqrt{\frac{\mu_{p} \bar{B} \epsilon_{H}}{M}}\left[\left(E(\kappa)-\left(1-\kappa^{2}\right) K(\kappa)\right]=\text { const } .\right.
$$

The averaged orbits of locally passing particles can be described by the invariant 


$$
J^{\text {pass }}=\psi_{p}-\frac{4 B_{3}}{\pi \omega_{B 0}} \frac{v_{\|}}{\left|v_{\|}\right|} \sqrt{\frac{\epsilon_{H} \mu_{p} \bar{B}}{M}} \kappa E\left(\kappa^{-1}\right)=\text { const. }
$$

\section{ORBITS OF FAST IONS IN W7-X}

A number of simplifying assumptions were used in the analysis made in the previous section. Therefore, direct numerical calculations of the particle orbits in realistic equilibria are required in order to see whether the conclusions drawn are true. This will be done below, where we study the fast ion behaviour in Wendelstein 7 -X by means of the code ORBIS. Wendelstein 7 -X will operate in various regimes with different magnetic configurations. We restrict ourselves to consideration of only two of them, the standard magnetic configuration with the central beta $\beta_{0}=6.8 \%$ and the high-mirror configuration with the same $\beta_{0}$. As follows from Fig. 1, the main difference between these configurations is that the mirror harmonic in the high-mirror case is larger by a factor of 3 in the core region and 2 at the periphery. In both configurations the mirror harmonic dominates in the plasma core, but $\epsilon_{m} \lesssim \epsilon_{h}$ at the plasma periphery in the standard configuration. The rotational transform in both cases is close to unity: $\iota_{0} \approx 0.86, \iota_{a} \lesssim 1$.

We found numerically that the particles that are well trapped in the local magnetic wells escape from the plasma with $E_{r}=0$ in the mentioned configurations of W7-X (this agrees with our consideration above: the parameter $\delta_{1} \approx 1.2$ for $\kappa \ll 1$ in the high-mirror configuration with $\beta_{0}=6.8 \%$, which is not sufficient to provide the confinement of welltrapped particles). Therefore, it is worth to consider a possibility to confine these particles by adding an electric field. According to Sec. III, the negative electric field is most appropriate for this purpose because then the electric-field-induced precession and the precession caused by plasma diamagnetism have the same directions. We assume that the electric field is localized around a certain radius, $r_{*}$, i.e., that there is an electric barrier. To select the magnitude of the electric field we use Eqs. (39), (41), which we write in a form convenient for the practical use as follows:

$$
\left.E_{r}\left(r_{*}\right) \gg \frac{\mathcal{E}_{k e V}}{r_{m}}\left(\epsilon_{h}-\epsilon_{t}\right)\right|_{r_{*}}\left(\frac{k V}{m}\right),
$$




$$
E_{r}\left(r_{*}\right)>\left.\frac{\mathcal{E}_{k e V}}{\Delta_{E, m}}\left(\epsilon_{h}-\epsilon_{t}\right)\right|_{r *}\left(\frac{k V}{m}\right),
$$

where $\mathcal{E}_{k e V}$ is the particle energy in $\mathrm{keV}, \Delta_{E, m}$ is a characteristic width of the electric transport barrier in metres, $r_{m}$ is $r$ in metres, and $\epsilon_{h}$ and $\epsilon_{t}$ in the considered W7-X configurations can be approximated as

$$
\epsilon_{h}=0.08 \frac{r}{a}, \quad \epsilon_{t}=0.05 \frac{r}{a} .
$$

Let us consider 20-keV protons (partly thermalized NBI particles or ICRH accelerated ions) in a plasma with the electric field characterized by $r_{*} / a=0.9$ and $\Delta_{E}=0.1 \mathrm{~m}$. Then Eq. (54) yields $E_{r}\left(r_{*}\right)>5.4 \mathrm{kV} / \mathrm{m}$, which also satisfies Eq. (53). Using this result, we calculated the particle orbits for various $E_{r}\left(r_{*}\right)$ exceeding $5.4 \mathrm{kV} / \mathrm{m}$ in the assumption that $E_{r}(r)=E_{*} \exp \left[-\left(r-r_{*}\right)^{2} / \Delta_{E}^{2}\right]$. We found that $E_{*}=5.8 \mathrm{kV} / \mathrm{m}$ is sufficient to provide the particle confinement in the high mirror configuration, see Fig. 3. In the standard configuration, the confinement was achieved with a somewhat higher electric field, $E_{*}=7$ $\mathrm{kV} / \mathrm{m}$.

Below we investigate a detrimental influence of the positive electric field on the particle confinement. We consider again the high mirror configuration but with an artificially decreased helical harmonic to satisfy Eq. (36). We found that due to this change of the magnetic configuration, the confinement of the well trapped particles in the plasma core is improved. We made calculations for the $50 \mathrm{keV}$ particles taking $\Omega_{E}=0$ and $\Omega_{E}(r)=$ const $<0$. It turned out that the particles, which are confined at $\Omega_{E}=0$, escape from the plasma when $\Omega_{E}$ satisfies Eq. (44), i.e. $\Omega_{E}^{r e s}=5766 \mathrm{~s}^{-1}$ (we used $\epsilon_{m}=0.1, \epsilon_{h}(a)=\epsilon_{t}(a)=\epsilon_{0}(a)=0.05$, $\left.a=50 \mathrm{~cm}, \rho=1.24 \mathrm{~cm}, \omega_{B}=2.5 \times 10^{8} \mathrm{~s}^{-1}\right)$. In addition, we found that particles escape from the plasma when $\Omega_{E}$ lies in a certain range depending on particle characteristics. In particular, particles with $\chi_{0}=-0.25 \varphi_{0}=170^{\circ}$ (the subscript " 0 " means that a quantity is taken at $t=0, \chi=v_{\|} / v$ is the pitch angle) and $r_{0} / a=0.5$ escape from the plasma when $3000 \mathrm{~s}^{-1}<\left|\Omega_{E}\right|<8200 \mathrm{~s}^{-1}$, whereas a particle with the same pitch angle but $r_{0} / a=0.3$ escapes from the plasma when $\Delta \Omega_{E} / \Omega_{\text {res }}=0.67$ and $3400 \mathrm{~s}^{-1}<\left|\Omega_{E}\right|<7300 \mathrm{~s}^{-1}$, see Fig. 4 .

In order to test Eq. (46) we considered a configuration with $\epsilon_{h}$ satisfying Eq. (55) but with an artificially increased diamagnetic harmonic, $\epsilon_{0}=0.127 r^{2} / a^{2}\left(\delta_{1}=6.3\right.$ for $\kappa \ll 1$ and $\left.E_{r}=0\right)$. In this modified configuration the well-localized particles are confined. We 
found that a particle with $r_{0} / a=0.3, \chi_{0}=0$ escapes from the plasma when $1.86 \times 10^{3} \mathrm{~s}^{1}<$ $\left|\Omega_{E}\right|<2.1 \times 10^{4} \mathrm{~s}^{1}$, see Fig. 5. Noting that $\Omega_{E}^{r e s}=1.4 \times 10^{4} \mathrm{~s}^{-1}$, we obtain $\Delta \Omega_{E} / \Omega_{\text {res }}=1.4$. On the other hand, Eq. (46) predicts $\Delta \Omega_{E} / \Omega_{E}^{r e s}=0.3 \delta_{1}$ which agrees with the numerical results when $\delta_{* 1}=4.7$. Note that this frequency range is much wider than that in the case of $\epsilon_{h}=\epsilon_{t}$ and corresponds to $2.4 \mathrm{kV} / \mathrm{m}<E_{r}(a)<27 \mathrm{kV} / \mathrm{m}$.

The calculations above are relevant to well-localized particles. Now consider another group of trapped particles, the transitioning particles. They have larger pitch angles and, therefore, are more easily produced by NBI in W7-X. When these particles are in the locally passing state, they oscillate along $\varphi$ with the amplitude $\Delta \varphi$, which exceeds the ripple period, $2 \pi / N$. Therefore, they have closed orbits in the $(r, \vartheta)$-plane, like orbits of trapped particles in tokamaks, even at $\beta=0$. For this reason, the electric field does not deteriorate their confinement. However, after transformation into the locally trapped state, these particles can escape from the plasma in the presence of the resonance electric field. Figure 6 demonstrates this for the high-mirror configuration and standard configuration.

It is of interest to see effects of the electric field by using the longitudinal invariant given by Eq. (51). With this purpose we calculate the contours of $J_{\|}=$const for the deeply trapped particles and moderately trapped particles in the W7-X high-mirror configuration. The results are shown in Fig. 7. It follows from Fig. 7(a) that a considerable fraction of particles is not confined. Adding a negative inhomogeneous electric field localized at $r_{*}=0.9 a$ makes $J$-contours in the vicinity of $r_{*}$ closed, which implies that particles are confined (Fig. 7(b)). Moderately trapped particles are confined in the absence of the electric field (Fig. 7(c)), whereas the presence of the positive electric field accompanied by the plasma rotation with the frequency about $\Omega_{E}^{r e s}$ deteriorates the confinement (Fig. $7(\mathrm{~d})$ ).

\section{ASH REMOVAL BY A POSITIVE ELECTRIC FIELD IN A HELIAS REACTOR}

The existence of the resonance electric field is not necessarily harmful. One can expect that this field may contribute to the removal of the ash (thermalized or partly thermalized $\alpha$-particles) in a fusion reactor. This may be the case when the electric field satisfies certain requirements. Because the difference between the thermalized alphas and bulk plasma ions is small from the point of view of sensitivity of their confinement to the radial electric field, we 
have to choose the electric field that removes only partly thermalized alphas. The magnitude of the required electric field can be evaluated by means of Eq. (48) with the particle energy in the range $T \ll \mathcal{E} \ll 3.5 \mathrm{MeV}$. In addition, the resonance must be sufficiently narrow in order to avoid a deterioration of the confinement of $\alpha$-particles in the $\mathrm{MeV}$ range and the bulk plasma ions.

We consider a five-period Helias reactor ${ }^{4}$ with $\bar{B}=5 \mathrm{~T}, a=2 \mathrm{~m}$. We take into account only dominant Fourier harmonics of the magnetic field using the following approximation: $\epsilon_{0}=0.08 r^{2} / a^{2}, \epsilon_{m}=0.1+0.02 r^{2} / a^{2}, \epsilon_{h}=0.08 r / a$, and $\epsilon_{t}=0.043 r / a$. Then we obtain from Eq. (48) that $\Omega_{E} \approx-240 \mathrm{~s}^{-1}$ for $\mathcal{E}^{r e s}=100 \mathrm{keV}$. The minimum energy of the particles lost because of the resonance electric field can be evaluated as $\mathcal{E}_{\min } \sim 50 \mathrm{keV}$ for $\delta_{* 1} \sim 3$, which follows from Eq. (50). One can think that the mentioned equation overestimates $\mathcal{E}_{\max }$ because its denominator approaches zero for $\delta_{* 1} \gtrsim 3$. Thus, direct numerical calculations are required. The results obtained by the code ORBIS for the localized particles, which start with various energies at a point on the $r=0.3 a$ flux surface, are shown in Fig. 8. We observe that particles with the energies $60 \lesssim \mathcal{E} \lesssim 170 \mathrm{keV}$ are lost, whereas particles with $\mathcal{E} \gtrsim 200 \mathrm{keV}$ and $\mathcal{E} \lesssim 50 \mathrm{keV}$ are confined.

Figure 8 demonstrates a possibility to satisfy the condition $T \ll \mathcal{E} \ll 3.5 \mathrm{MeV}$ for the lost alphas. However, the calculations carried out are not sufficient to conclude how many partly thermalized alphas can be removed from the reactor by the electric field. In addition, Fig. 8 was obtained in the assumption that $\Omega_{E}(r)=$ const, i.e., $E_{r} \propto r$. Calculations based on a more realistic radial profile of the electric field are required to conclude whether the electric field can really help to solve the problem of ash removal. On the other hand, we have to note that the effect of removal of the fast ions by the resonance electric field is relatively weakly dependent on the radial profile of this field because the resonance is rather wide.

\section{SUMMARY AND CONCLUSIONS}

The code ORBIS (ORBits In Stellarators) for the investigation of the particle orbital motion in stellarators is developed. The code solves equations of the particle guiding center motion in the stellarator magnetic field in Boozer coordinates in the presence of the radial electric field. To show the orbits of the confined particles and lost particles clearly, their motion since the birth moment is visualized. 
Bounce-averaged equations of the particle guiding center motion are derived and used for a qualitative analysis of the influence of the electric field on the orbits of trapped particles in systems with a dominant or considerable mirror harmonic of the magnetic field, which is the case in the Wendelstein-line stellarators.

The results obtained by means of an analysis of the bounce-averaged equations and numerical study of the particle orbits by the code ORBIS in Wendelstein 7-X and a Helias reactor can be summarized as follows.

(i) The presence of the negative electric field tends to improve the trapped ion confinement. The particles are confined by the electric field when their energy does not exceed a certain magnitude. An electric field localized in a ring region can play the role of a transport barrier for the energetic ions.

(ii) The positive electric field deteriorates the confinement of trapped ions, unless the magnitude of the electric field is very large. A detrimental influence of the positive electric field is especially strong when it leads to a rigid plasma rotation with the frequency that satisfies a certain condition, which we refer to as a resonance condition. The resonance rotation frequency, $\Omega_{E}^{r e s}$, is a function of the particle energy, $\mathcal{E}$. When $\Omega_{E}=\Omega_{E}^{r e s}$, welltrapped particles with a certain energy escape from the plasma. The resonance can be rather wide, i.e., the range of rotation frequencies, $\Delta \Omega_{E}$ around $\Omega_{E}^{r e s}$ for which particles with the given energy are not confined can be large. When this is the case, the effect of the $E$-field induced loss of the fast ions is robust, i.e., the effect exists for $\Omega_{E}^{r e s}$ which arbitrary varies with radius around $\Omega_{E}^{\text {res }}$ in a certain range $\Delta \Omega_{E}^{\text {res }}$.

On the other hand, plasma rotation associated with the positive electric field can lead to the loss of well-trapped particles with the energies in a certain range, $\Delta \mathcal{E}$.

(iii) The confinement of the transitioning particles is also affected by the radial electric field. Because of this, a theory of stochastic diffusion (i.e. the collisionless diffusion of transitioning particles ${ }^{9}$, which is presumably responsible for the main channel of loss of fast ions in the Wendelstein-line stellarators) should be generalized to include effects of the electric field.

(iv) A positive electric field satisfying the resonance condition for ions with the energy in the range $T \ll \mathcal{E} \ll 3.5 \mathrm{MeV}$ will remove partly thermalized trapped $\alpha$-particles (ash) from the plasma in the Helias reactor. As a result, a loss cone in the alpha velocity distribution will be formed in the resonance region. Owing to this, in addition to trapped particles, 
partly slowed down circulating alphas can be removed. This will be the case when $\mathcal{E}^{\text {res }} \lesssim$ $\left(M_{i} / M_{e}\right)^{1 / 3} T$, because then the Coulomb pitch-angle scattering will be comparable to slowing down. In addition, plasma instabilities driven by the velocity anisotropy of alphas can arise and contribute to the ash removal. Thus, there is a hope that the radial electric field will help to solve the problem of ash removal in the Helias reactor. Further investigation is required in order to make a more definite conclusion. A key question is whether transport processes in fusion plasmas will generate and sustain the required electric field. Another important question is whether the considered mechanism is effective, i.e., whether it will provide the removal of most partly thermalized $\alpha$-particles.

\section{ACKNOWLEDGMENTS}

The work is carried out within the Partner Project Agreement P-034e between Science and Technology Center in Ukraine, Institute for Nuclear Research, and Max-Planck-Institut für Plasmaphysik. The authors thank Yu.V. Yakovenko for his comments.

1 G. Grieger and the W7-X Team, J. Plasma Fusion Res. SERIES 1, 53 (1998).

2 M. Wanner, V. Erckmann, J.-H. Feist et al., Nucl. Fusion 43, 416 (2003).

3 A. Kreter, J. Baldzuhn, V.I. Davydenko et al., http://www.dpgtagungen.de/archive/2003/p_4.html

4 J. Kisslinger et al., 1998 Fusion energy Proc. 17th Int. Conf. (Yokohama, 1998) vol 4, (Vienna: IAEA) 1239 (1999).

5 W. Lotz, P. Merkel, J. Nührenberg, E. Strumberger, Plasma Phys. Contr. Fusion 34, 1037 (1992).

6 J. Baldzuhn, A. Werner, H. Wobig et al., Plasma Phys. Contr. Fusion 45891 (2003).

7 C.D. Beidler, H. Maaßberg "Implications of the Quasi-Neutrality Condition for Neoclassical Transport in Stellarators", a report in the IAEA TM on Innovative Concepts and Theory of Stellarators, Madrid 2005.

8 H. Wobig, private communication.

9 C.D. Beidler, Ya.I. Kolesnichenko, V.S. Marchenko, I.N. Sidorenko, H. Wobig, Phys. Plasmas 
8, $2731(2001)$.

10 A.A. Galeev, R.Z. Sagdeev, "Theory of Neoclassical Diffusion", in Reviews of Plasma Theory, Vol. 7 (Consultants Bureau, New York - London, 1979), p. 257-343.

11 K. Itoh K, S-I. Itoh, Plasma Phys. Contr. Fusion 38, 1 (1996). 
Figures

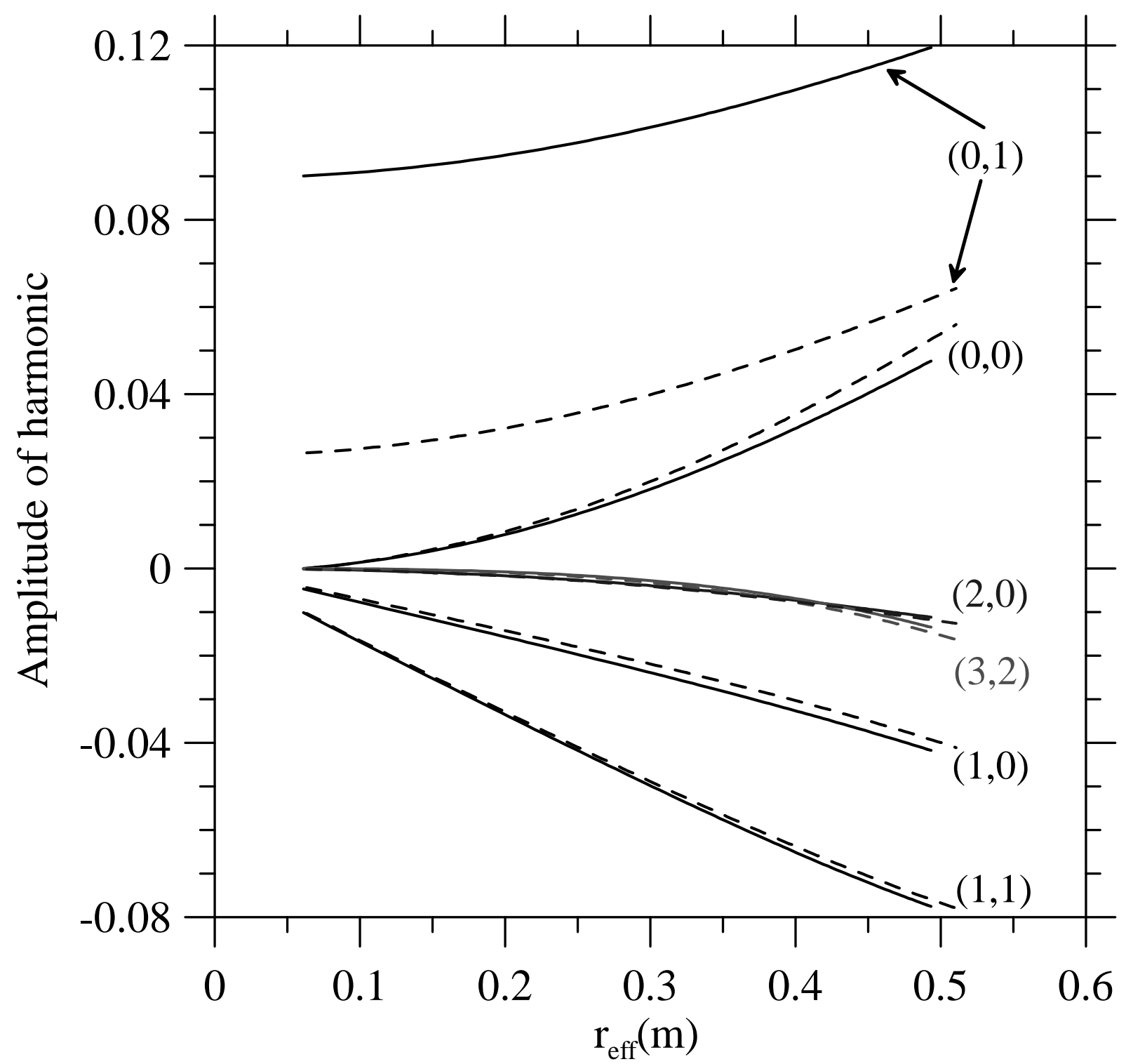

FIG. 1: Main Fourier harmonics, $\epsilon_{B}^{(\mu \nu)}$, of the magnetic field in W7-X with $\beta(0)=6.8 \%$ : solid lines correspond to the high-mirror configuration, dashed lines correspond to the standard configuration. 


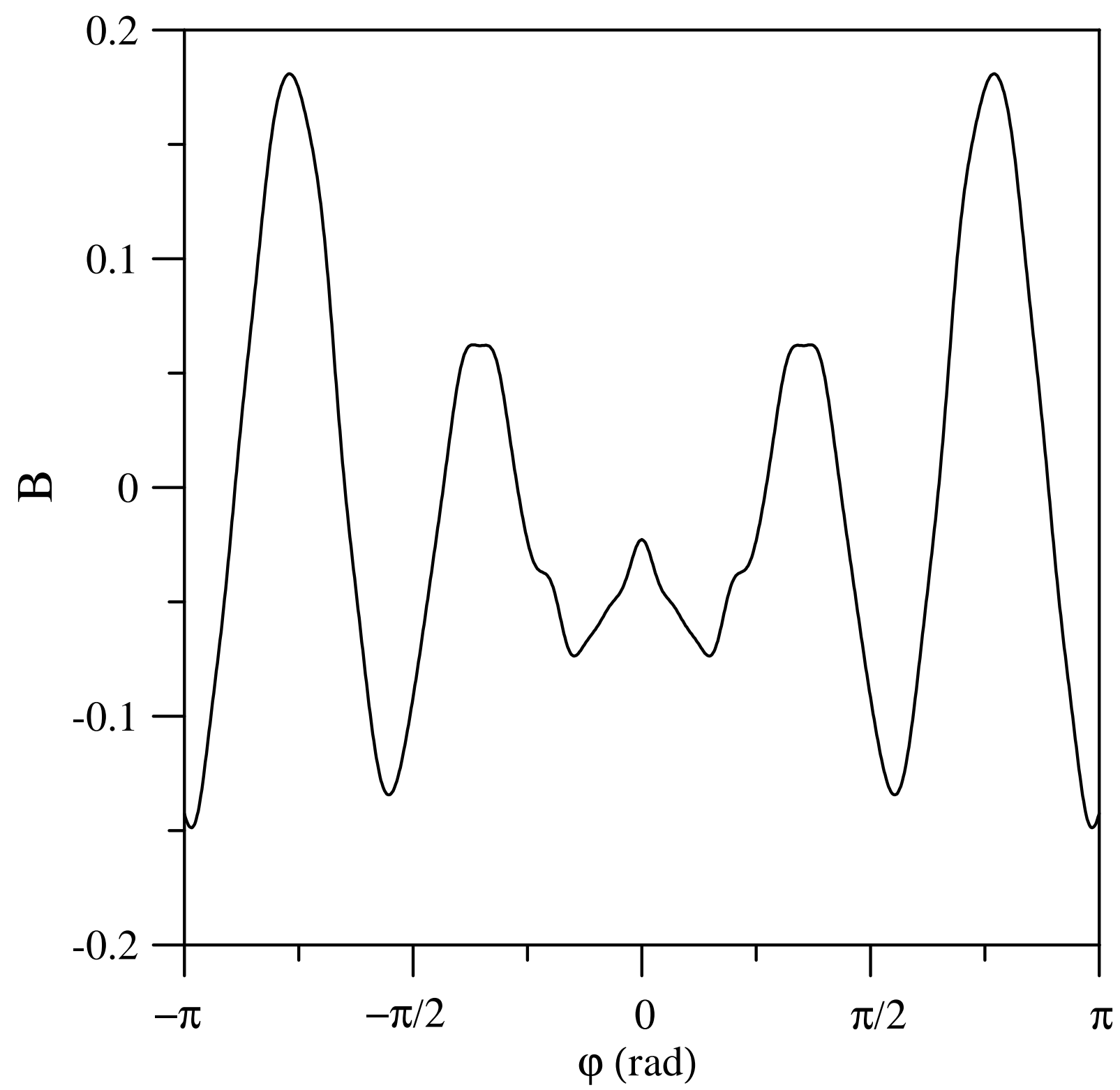

FIG. 2: The magnetic field strength along the field line going through the point $r=a / 2, \vartheta=0$, $\varphi=0$ in the W7-X standard configuration. The maximum at $\varphi=0$ is rather small, therefore, small-pitch-angles particles produced by NBI or IRCH at this angle are transitioning. 


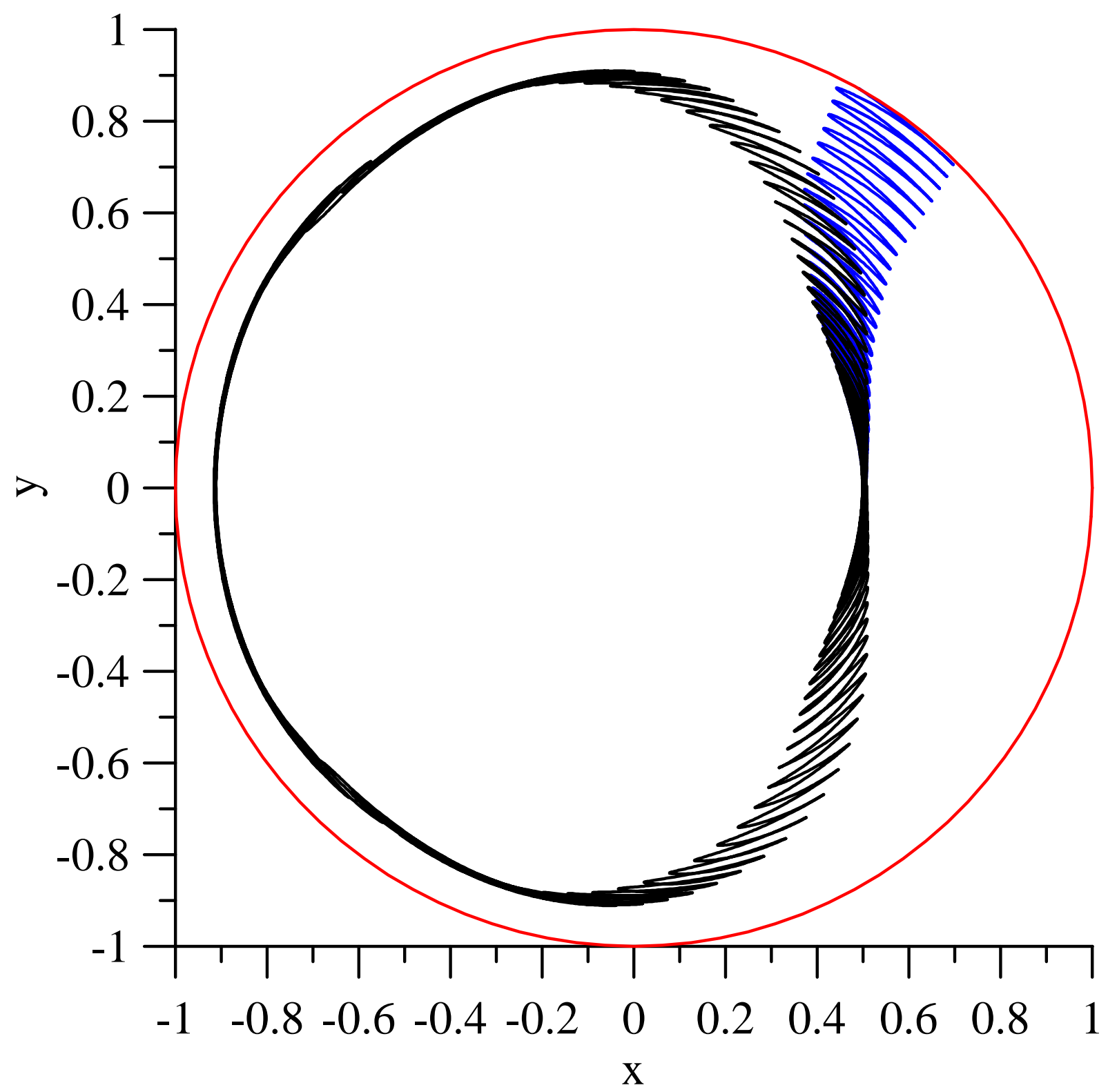

FIG. 3: Motion of a localized 20-keV proton in the poloidal cross section of the W7-X high-mirror configuration with $E_{r}=0$ (lost particle) and in the presence of the negative electric barrier, $E(r)=-E_{*} e^{-\left(r-r_{*}\right)^{2} / \Delta_{E}^{2}}$ with $E_{*}=5.8 \mathrm{kV} / \mathrm{m}, r_{*} / a=0.9, \Delta_{E}=0.1 \mathrm{~m}$. The starting point is $r_{0} / a=0.5, \vartheta_{0}=0, \varphi_{0}=170^{\circ}$, where $\chi_{0}=0.1$. The particle quickly escape from the plasma with $E_{r}=0$, but it is confined in the presence of the electric barrier. 

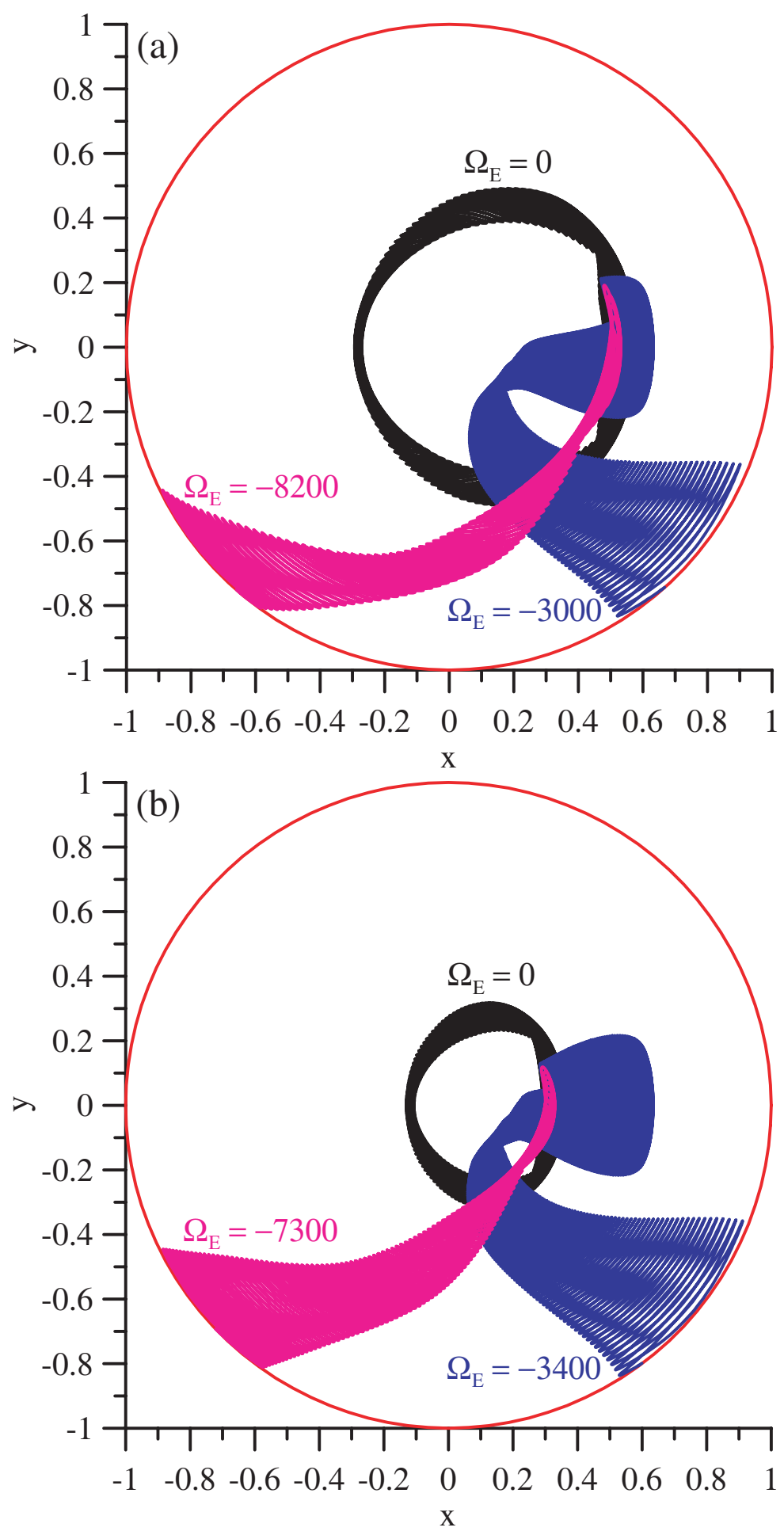

FIG. 4: Motion of a localized $50-\mathrm{keV}$ proton in the poloidal cross-section of W7-X high-mirror configuration with the decreased helical harmonic $\epsilon_{h}\left(\epsilon_{h} \rightarrow \epsilon_{h}-0.03 r^{2} / a^{2}\right)$ in the absence of electric field and for different $\Omega_{E}: \mathrm{a}, r_{0} / a=0.5 ; \mathrm{b}, r_{0} / a=0.3$. At the starting point $\vartheta_{0}=0$, $\varphi_{0}=170^{\circ}$, and $\chi_{0}=-0.25$. The electric field leads to the particle loss. 


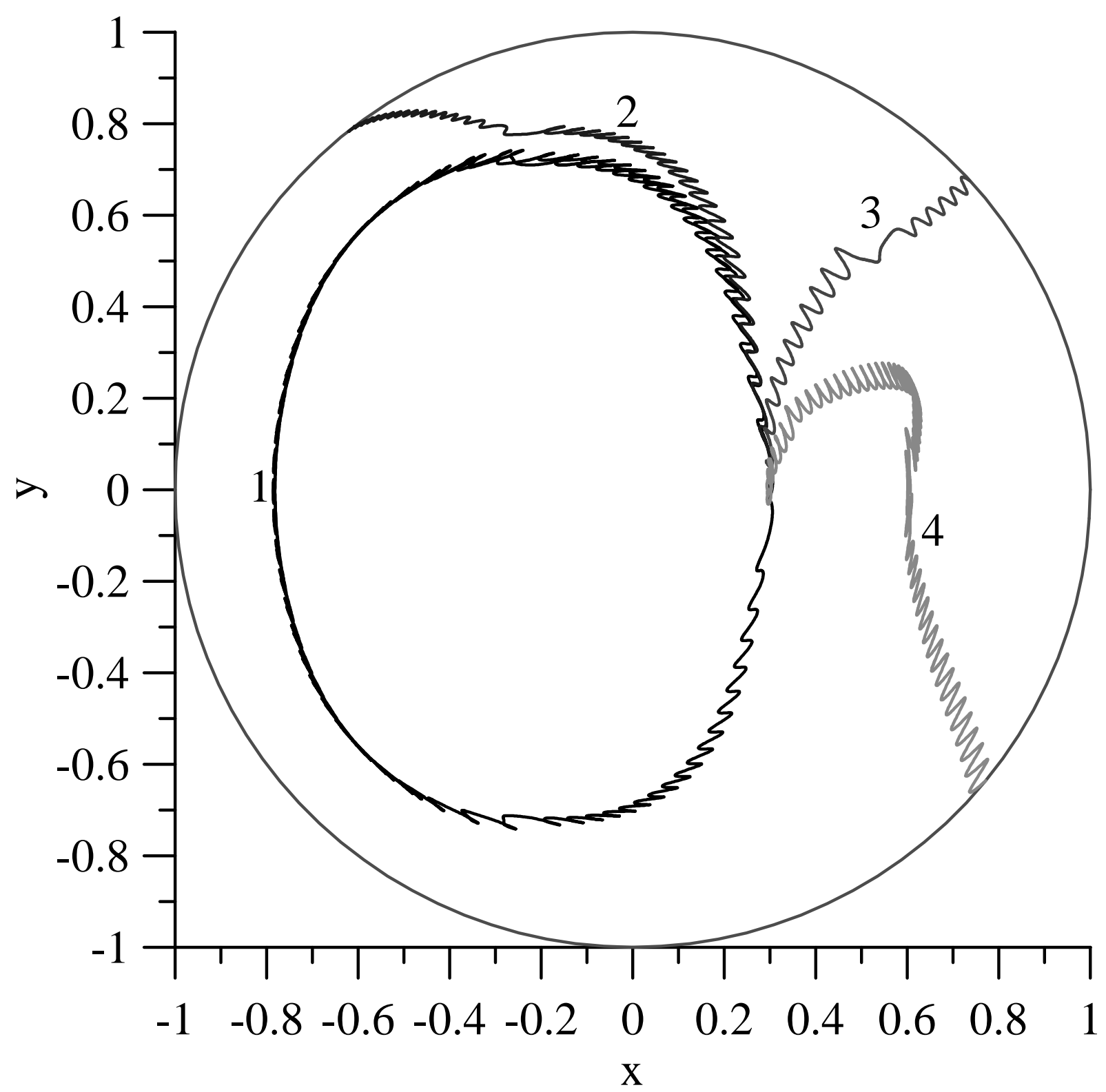

FIG. 5: Motion of a localized 50-keV proton in the poloidal cross-section of W7-X high-mirror configuration with high- $\beta$ at $E_{r}=0$ (curve 1) and in the presence of the resonance electric field $\left(E_{r}>0\right)$ determined by $\Omega_{E}=-1.86 \times 10^{3} \mathrm{~s}^{-1}$ (curve 2), $\Omega_{E}=-1.3 \times 10^{4} \mathrm{~s}^{-1}$ (curve 3), and $\Omega_{E}=-2.1 \times 10^{4} \mathrm{~s}^{-1}$ (curve 4). At the starting point $r_{0} / a=0.3, \vartheta_{0}=0, \varphi_{0}=41^{\circ}$, and $\chi_{0}=0$. 

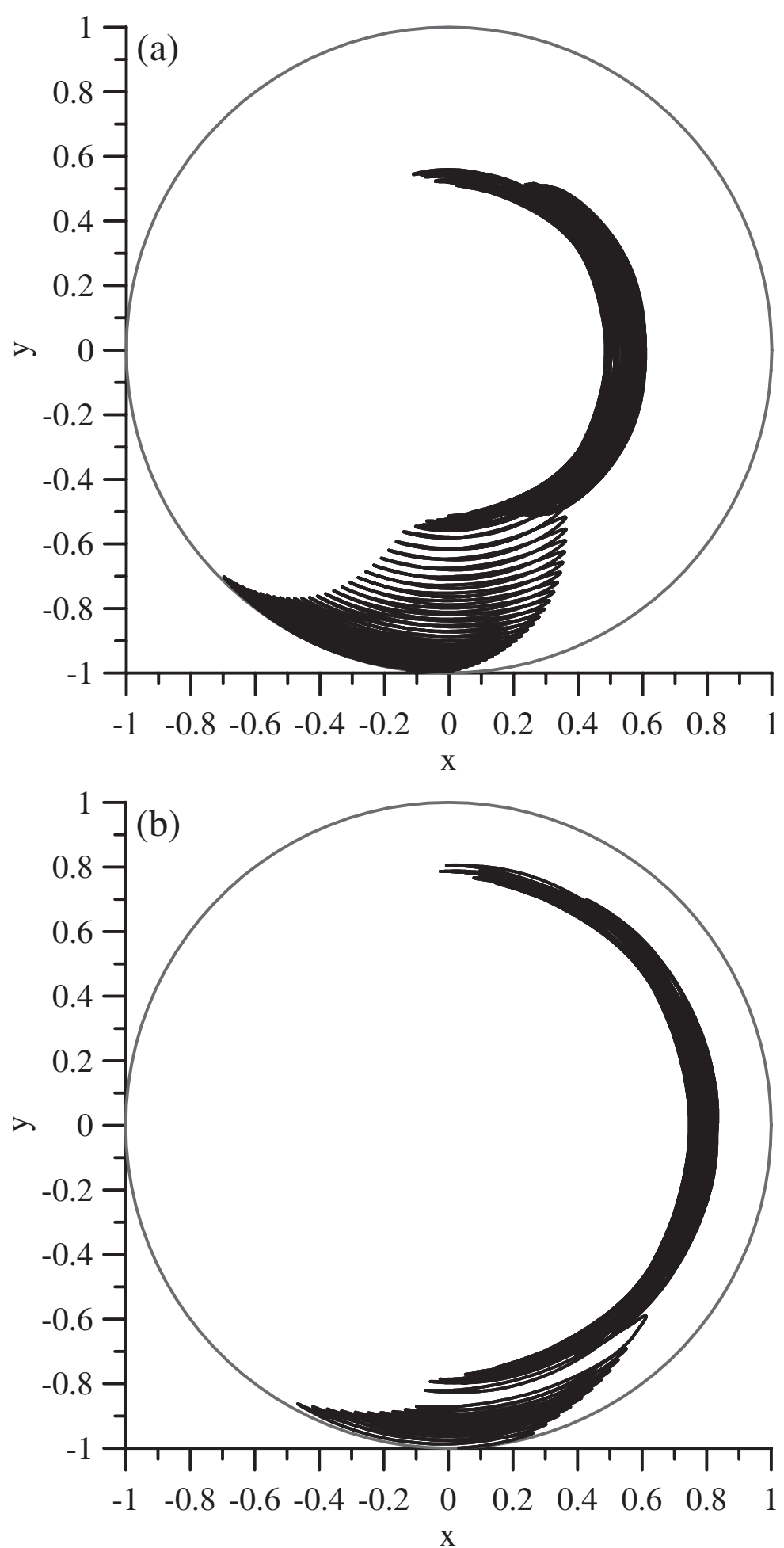

FIG. 6: Motion of locally passing 20-keV protons in the poloidal cross-section of $\mathrm{W} 7-\mathrm{X}$ in the presence the resonance electric field determined by $\Omega_{E}=-4 \times 10^{3} \mathrm{~s}^{-1}, \vartheta_{0}=0, \varphi_{0}=170^{\circ}$ : a, high-mirror configuration, $r_{0} / a=0.5, \chi_{0}=-0.35$; b, standard configuration $r_{0} / a=0.75$, $\chi_{0}=-0.25$. The particle is lost after it is transformed into a localized particle. The considered particles are confined in the absence of the electric field. 

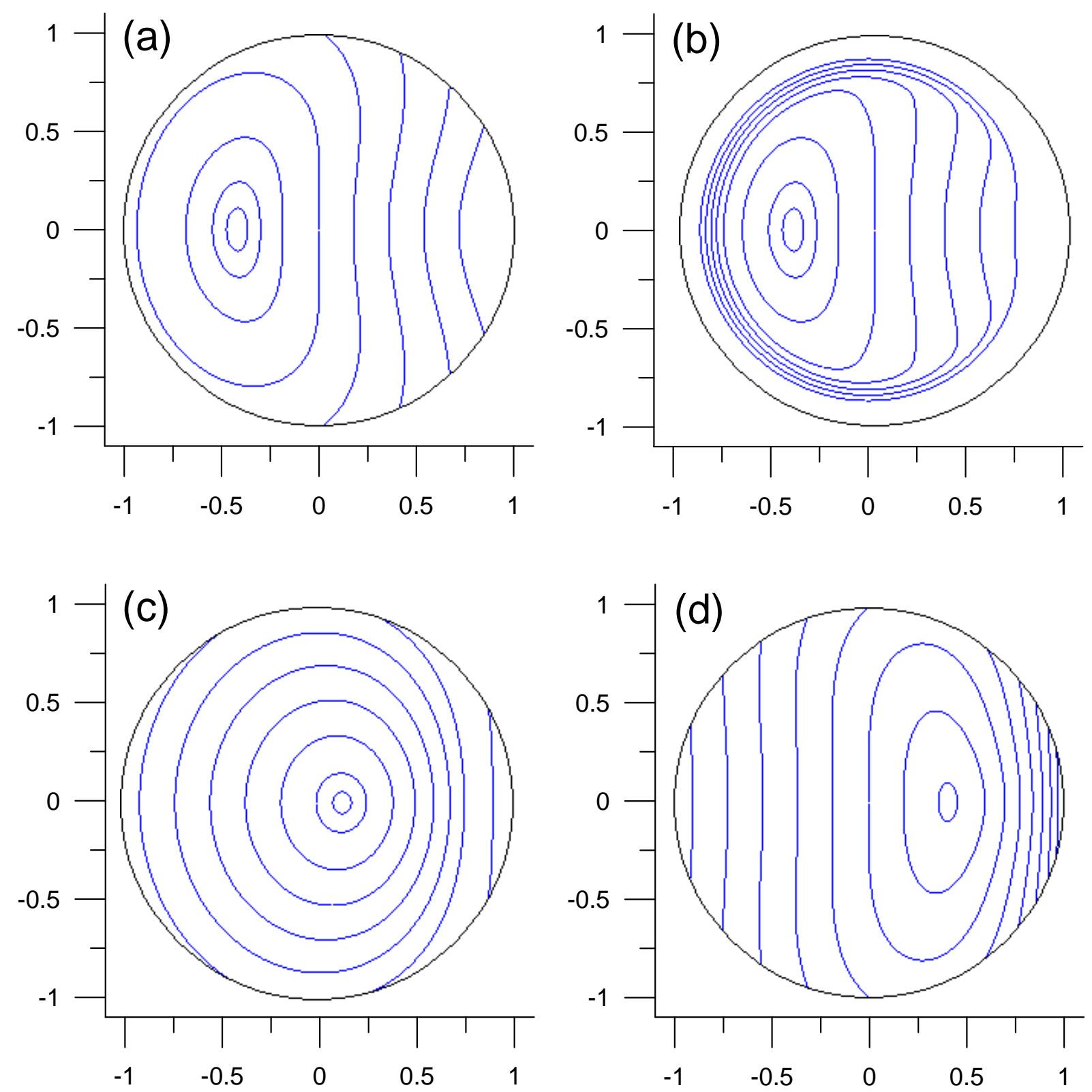

FIG. 7: Contours of $J_{\|}=$const of the $\mathcal{E}=50-\mathrm{keV}$ protons in W7-X: a, deeply trapped particles with $\alpha=-0.05$ at $E_{r}=0$; b, the same particles in the presence of the electric transport barrier, $E(r)=-E_{*} e^{-\left(r-r_{*}\right)^{2} / \Delta_{E}^{2}}$ with $E_{*}=5.8 \mathrm{kV} / \mathrm{m}, r_{*} / a=0.9, \Delta_{E}=0.1 \mathrm{~m} ; \mathrm{c}$, moderately trapped particles with $\alpha=0$ at $E_{r}=0$; d, the same particles that in the case "c" but when $\left|\Omega_{E}\right|=$ $8 \times 10^{-3} \mathrm{~s}^{-1}, E_{r}>0$. The calculations are based on Eq. (51). 


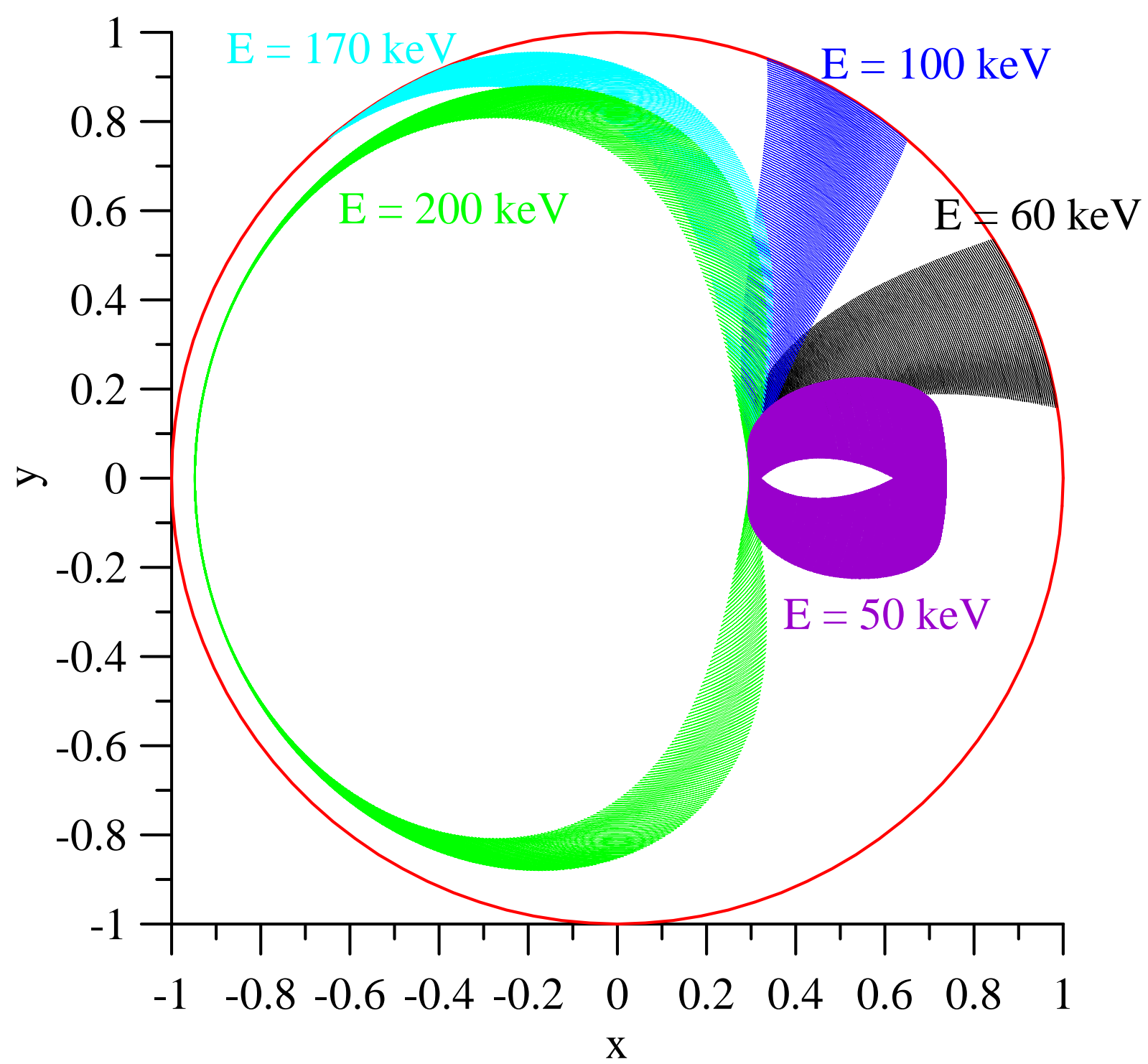

FIG. 8: Motion of partly thermalized $\alpha$-particles in a five-period Helias reactor with $\Omega_{E}=$ $-240 \mathrm{~s}^{-1}$. All the considered particles start at the point $r_{0} / a=0.3, \vartheta_{0}=0, \varphi_{0}=36^{\circ}$, where they have the pitch angle $\chi_{0} \equiv v_{\|} / v=0.2$. 